\title{
THERMOACOUSTIC STIRLING ENGINE WITH LIQUID PISTON
}

\author{
H. Hyodo ${ }^{1 *}$, R.Uchiya ${ }^{1}$, and T. Biwa ${ }^{1}$ \\ ${ }^{1}$ Mechanical Systems and Design, Tohoku University, 6-6-04 Aramaki, Aoba-ku, 980-8579 Sendai, Japan. \\ *Corresponding author's e-mail: hyodo@amsd.mech.tohoku.ac.jp
}

Keywords: modeling of thermoacoustic engine, liquid column piston.

\section{Introduction}

The thermoacoustic engine with liquid piston was proposed by West [1] and confirmed its operation by Martini [2] and Li [3]. However, the effects of parameters such as number of liquid column and length of it on the operation of the devices are not discussed. In this study, by modelling the looped thermoacoustic engines with gas-liquid column, we clarify the basic mechanism of the engine.

\section{Analytical model}

The thermoacoustic engine with gas-liquid column is modeled by the spring-mass system to examine the characteristic oscillation mode. The liquid column, gas column and effect of gravity force in thermoacoustic engines are replaced with mass point (mass: $m$ ), spring (spring constant: $k_{a}$ ) and elastic rod (spring constant: $k_{g}$ ), respectively as shown in figure 1.

(a)

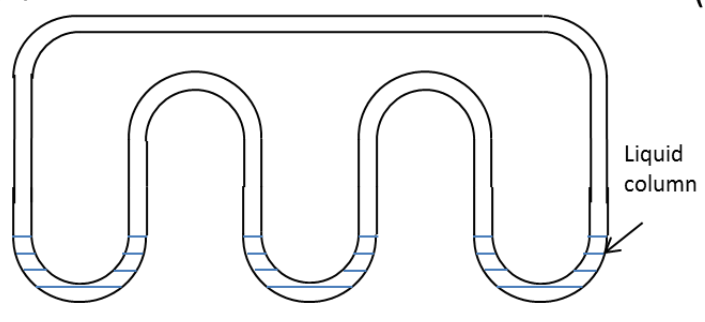

(b)

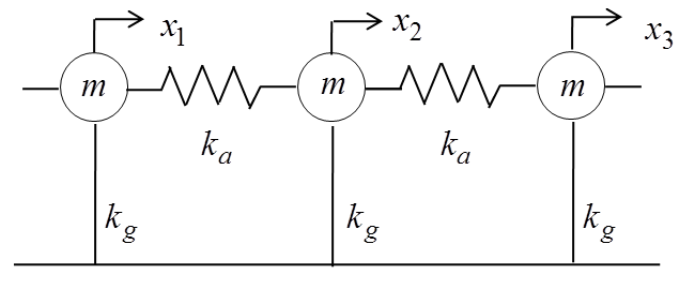

Figure 1: (a) Liquid piston engine (b) Spring-mass system model.

The constant $k_{a}$ and $k_{g}$ are assumed to be $k_{a}=A P_{m} \gamma / l_{g a s}, k_{g}=2 A \rho_{w} g$, where $A$ is the cross sectional area of the tube, $P_{m}$ is the mean pressure of the gas, $\gamma$ is the ratio of specific heat, $l_{g a s}$ is the length of the gas column, $\rho_{\mathrm{w}}$ is the density of the gas and $g$ is the gravity acceleration. When the number of liquid column is $n$, the equations of motion of this system is expressed by

$$
m \ddot{x}_{j}=-k_{g} x_{j}-k_{a}\left(x_{j}-x_{j+1}\right)-k_{a}\left(x_{j}-x_{j-1}\right)
$$

where $x_{j}(j=1, \ldots n)$ is the displacement of mass. We focus on the solution that the phase of the complex amplitude of $x_{j}$ is given by $2 \pi j / \mathrm{n}$, whereas the magnitude satisfies $x_{j}=$ const. In this case, frequency equation is written as

$$
\omega^{2}=\frac{k_{g}+2 k_{a}\left(1-\cos \frac{2 \pi}{n}\right)}{m} .
$$

The pressure $p_{j}$ and velocity $u_{j}$ of the gas in the middle of tube between the water column are given by $p_{j}=k_{a}\left(v_{j-1}-v_{j}\right) / \mathrm{i} \omega A$ and $u_{j}=\left(v_{j-1}+v_{j}\right) / 2$, where $v_{j}$ is the velocity of mass given by $v_{j}=\mathrm{d} x_{j} / \mathrm{d} t$. 
Calculating the acoustic impedance from pressure and velocity of gas, we obtain the impedance $z_{j}$

$$
Z_{j}=\frac{2 k_{a}}{\omega A} \tan \frac{\pi}{n}
$$

In equation (3), the acoustic impedance is real, which means the pressure and velocity oscillate in phase. Then if regenerator is put at this point, Stirling cycle should be realized regardless to the number of liquid columns.

\section{Calculation by thermoacoustic theory}

We calculate further details in more realistic model including regenerator and temperature gradient by using transmittance matrices based on Rott's theory. We consider a looped tube engine in which the regenerators are inserted into the center of the gas column tubes. The apparatus consists of the same shaped three sections as shown in fig2 (a). Setting the parameters as fig 2(a) and imposing periodic boundary condition, marginal conditions of this engine was derived shown in fig 2(b). Fig 2(b) shows the relation of pore radius of regenerator and angular frequency and temperature ratio of hot and cold heat exchangers. The data of dots were results calculated by thermoacoustic theory and dotted line in the graph of frequency was calculated by the equation of spring-mass model. The oscillation frequencies calculated by thermoacoustic theory were around 43, these results agree well with that of spring-mass model. We experimentally confirmed the operation of the apparatus at predicted frequency. This means that the system operates in the characteristic oscillation mode we focused on in the mass-spring model.

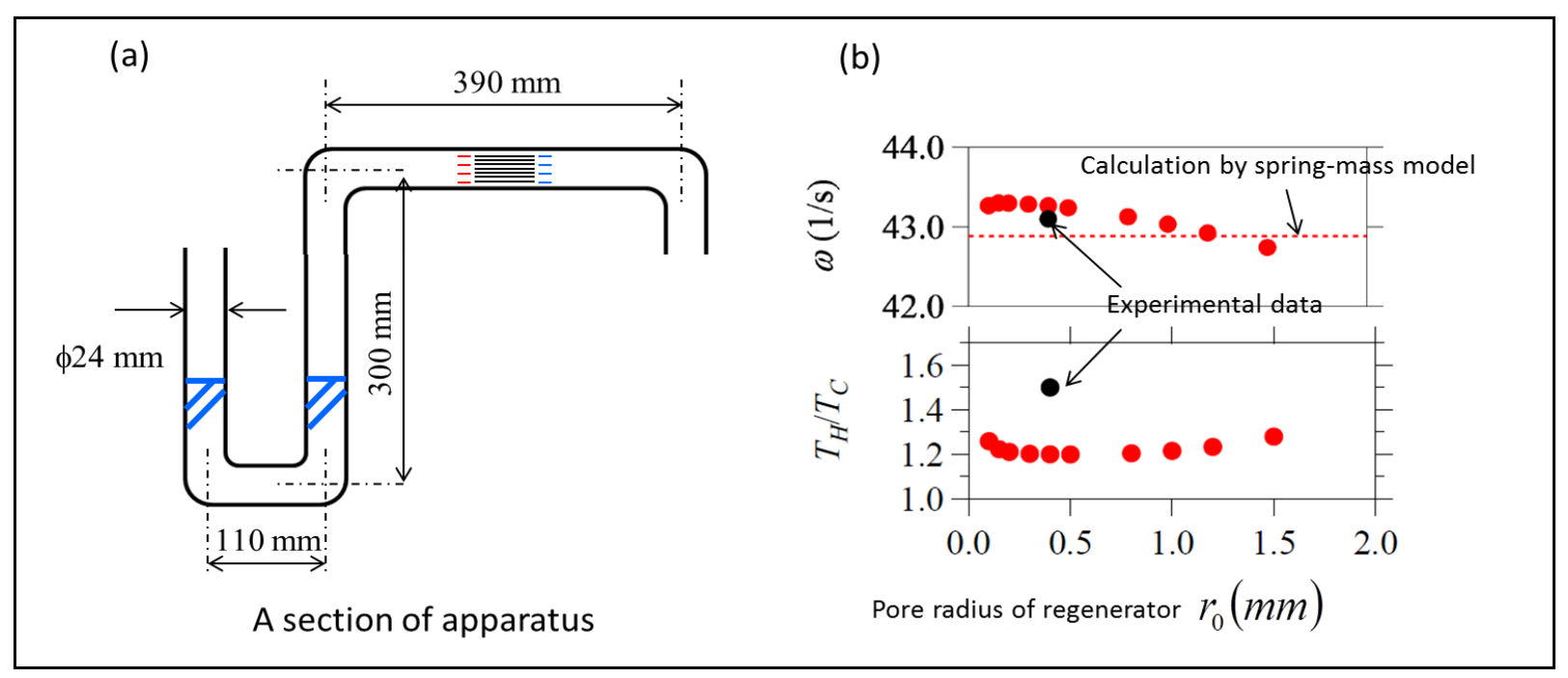

Figure 2: (a) Parameters of the apparatus (b) Oscillation marginal conditions.

\section{Summary}

We modeled the thermoacoustic engines with liquid piston by the spring-mass system. The characteristic oscillation mode of the system became obvious and Stirling cycle should be realized.

\section{References}

[1] West, C.D. “Liquid piston stirling engines”, Van Nostrand Reinhold, (1983)

[2] Martini, W. R. "Test on a 4 u tube heat operated heat pump" Proc., Intersoc. Energy Convers. Eng. Conf., 2, (1983), 872-874.

[3] Li, D., Luo, E., Chen, Y., Zhang, L., and Huang, Y. "A looped three-stage traveling-wave thermoacoustic engine with liquid piston", 19th International Congress on Sound and Vibration, (2012) 